\title{
Influencia de las tendencias del comportamiento del consumidor turístico en el desarrollo responsable del destino Holguín
}

\author{
Influence of tourist consumer behavior trends in the responsible \\ development of the Holguin destination
}

Lic. Elizabeth del Carmen Pérez Ricardo. ${ }^{1}$, MSc. Justa Ramona Medina Labrada. ${ }^{2} \&$ Lic. Noel Fernández Cueria. ${ }^{3}$

\begin{abstract}
New trends in tourist consumer behavior have changed the way business operates internationally. Today, customers are loyal to brands that care about preserving the planet, local communities, and society in general. In this way, companies seek to respond to the growing demands of consumers, assuming social responsibility policies, understood as a new business culture based on ethical management, which can serve as a channel to improve the competitiveness and reputation of their businesses. Responsible tourism emerges in this context, which tries to offer an enriching experience to the client, at the same time that it corresponds to the new trends in consumer behavior. This type of tourism not only allows organizations to differentiate themselves in the market, but also to show a more humane image and obtain a reputation as a socially responsible tourism company, which can be an important competitive advantage. Holguin destination, of great relevance to the tourism industry in the country's east, has opted for responsible tourism as a development strategy, for which reason this research aims to assess the influence of international trends in tourist consumer behavior in the development of responsible tourism in the destination. To identify the main trends in the behavior of the tourist consumer from the literature consulted, a correlation analysis was performed and, in addition, expert judgment was used to determine the degree of influence of these trends in the development of responsible tourism. As a result, those trends in consumer behavior that influence good practices in responsible tourism in the destination Holguín were identified and the indicators that have positive results in the destination and the indicators that should be worked to achieve a total practice of tourism were analyzed. responsable.
\end{abstract}

Key Words: Trends, Consumer Behavior, Responsible Tourism, Tourist Destination.

\footnotetext{
1 elizabeth.ricardo@uho.edu.cu

2 jmedina@uho.edu.cu
} 


\section{Resumen}

Las nuevas tendencias del comportamiento del consumidor turístico han modificado la forma de operar de los negocios a nivel internacional. Actualmente, los clientes son fieles a las marcas que se preocupan por la preservación del planeta, las comunidades locales y la sociedad en general. De esta manera, las empresas buscan responder a las crecientes demandas de los consumidores, asumiendo políticas de responsabilidad social, entendidas como una nueva cultura empresarial basada en la gestión ética, que puede servir como cauce para mejorar la competitividad y la reputación de sus negocios. En este contexto emerge el turismo responsable, que trata de ofrecer una experiencia enriquecedora al cliente, al mismo tiempo que se corresponde con las nuevas tendencias en el comportamiento de los consumidores. Esta modalidad de turismo no sólo permite a las organizaciones diferenciarse en el mercado, sino mostrar una imagen más humana y obtener una reputación de empresa turística socialmente responsable, lo cual puede constituir una importante ventaja competitiva. El destino Holguín, de gran relevancia para la industria turística en el Oriente del país, ha apostado por el turismo responsable como estrategia de desarrollo, por lo cual la presente investigación tiene como objetivo evaluar la influencia de las tendencias internacionales del comportamiento del consumidor turístico en el desarrollo del turismo responsable en el destino. Para identificar las principales tendencias del comportamiento del consumidor turístico a partir de las literaturas consultadas fue realizado un análisis de correlación y además se empleó el criterio de expertos para determinar el grado de influencia de estas tendencias en el desarrollo del turismo responsable. Como resultado se identificaron aquellas tendencias del comportamiento del consumidor que influyen en las buenas prácticas del turismo responsable en el destino Holguín y se analizaron los indicadores que poseen resultados positivos en el destino y los indicadores en que se debe trabajar para alcanzar una práctica total de turismo responsable.

Palabras Clave: Tendencias, Comportamiento del Consumidor, Turismo Responsable, Destino Turístico.

\section{Introducción}

Cada vez son más numerosas las organizaciones, gubernamentales y no gubernamentales, lucrativas y sin ánimo de lucro, académicas, privadas y públicas, así como diferentes movimientos sociales y expertos, los que se están acercando a este enfoque del "turismo sostenible y responsable". En la última década particularmente se ha venido adoptando una actitud cada vez más crítica con respecto a los modelos turísticos masivos y desarrollistas imperantes a nivel internacional, no solamente en el ámbito académico y científico sino también, aunque de manera más tímida, en el sector empresarial y profesional del turismo e incluso en la esfera de las administraciones públicas con incidencia directa o indirecta en el desarrollo de la actividad turística (Rivera Mateus y Rodríguez García, 2012).

Estas nuevas ópticas están integrándose en el concepto paraguas de turismo responsable, asociado, a su vez, a otras denominaciones y formas de hacer y entender el turismo como 
el turismo solidario, el turismo ético, el ecoturismo, el turismo étnico, el turismo intercultural, el turismo comunitario o el turismo como instrumento de lucha contra la pobreza. La OMT en 2009 definió el turismo responsable como la "actividad turística que los turistas y los agentes turísticos realizan con respeto por los lugares de acogida desde el punto de vista medioambiental, cultural, social y económico, y que a su vez la comunidad receptora acepta y respeta y las autoridades públicas protegen y fomentan", todo ello de acuerdo con los principios recogidos en el Código Ético Mundial del Turismo. Por tanto, el turismo responsable trata de atender a las comunidades locales y de estar cerca de ellas, distribuyendo los beneficios de la industria de manera más equitativa y amplia hacia toda la población.

Por otra parte, la expansión del consumo responsable, en sus distintas modalidades, es un factor que incentiva a las empresas a adoptar prácticas de responsabilidad social corporativa. De esta manera, existe una tendencia creciente a que la sociedad y los consumidores exijan a la empresa conductas cada vez más responsables y transparentes (Ruano y Rojas, 2006). A su vez, el éxito de las empresas está de alguna manera relacionado con el éxito de las comunidades en las que se desenvuelven, lo cual es relevante en el caso de las empresas turísticas, cuyo éxito depende, en gran medida, del atractivo global de los destinos turísticos en los que se localizan, funcionando como un sistema.

Pero más allá de las iniciativas emprendidas hasta ahora, en su mayor parte aisladas o de limitada incidencia, las respuestas han de venir del lado del impulso de las capacidades e iniciativas locales, tanto públicas como privadas y en la articulación de estrategias adecuadas y decididas por parte de los gobiernos, capaces de implicar a todos los agentes sociales y económicos en la definición de proyectos endógenos, auto centrados y de turismo comunitario que puedan contribuir a invertir la actual proporción costos/beneficios del sector y minimizar en el territorio sus impactos negativos.

Dada la posición del turismo como uno de los primeros sectores de exportación y creación de empleo, la OMT defiende la necesidad de un crecimiento responsable. El turismo ocupa, por lo tanto, un lugar primordial en las políticas mundiales de desarrollo, y puede aún alcanzar un mayor reconocimiento político e incidir de manera efectiva en este "decenio de acción", con solo diez años por delante para alcanzar la Agenda 2030 y sus 17 Objetivos de Desarrollo Sostenible (OMT, 2020).

El turismo responsable, por sí sólo, puede ser una parte de esa alternativa, necesitando, además, alinearse con la pequeña y mediana empresa turística local y nacional para permitir un desarrollo turístico de carácter endógeno. Se favorece de esta manera la oportunidad para un cambio de los modelos turísticos tradicionales, tan cuestionados por la crisis económica actual, en la forma de ver el turismo. Asimismo, resulta necesario que todo el sector turístico y los propios consumidores en general asuman que el turismo responsable, al igual que el turismo sostenible, no es sólo un producto alternativo más destinado a una minoría de turistas más sensibilizados de los países desarrollados, sino que va más allá al tratarse de un compromiso y una forma diferente de hacer turismo, que 
debe ir impregnando poco a poco a todos los subsectores de producción turística, a los destinos y a la gobernanza turística en el ámbito internacional. Se refiere, entonces, a la adopción en el sector turístico de "una filosofía de pensar globalmente y actuar localmente" (Rivera Mateus y Rodríguez García, 2012).

A su vez, es necesario impulsar procesos que refuercen la capacidad innovadora de los destinos, procesos adaptados a su situación real, a los recursos y capacidades tanto de los agentes públicos como privados. En función de esto se debe tener en cuenta que los destinos de avanzada los diseña la comunidad, demuestran sensibilidad y responsabilidad ambiental, son capaces de crear ventajas competitivas, están comprometidos con la cohesión y el desarrollo social, se basan en estructuras coherentes de gobernanza, se muestran dialogantes con el entorno, persiguen la innovación, establecen conexiones con redes de ciudades, utilizan tecnología digital e integran el mundo físico y el virtual (López et al., 2015). En este contexto de cambio, la gestión turística local no puede permanecer invariable, de manera que la gestión turística tradicional, que venía acusando serios problemas de eficiencia, incrementa su grado de obsolescencia y hace más evidente la necesidad de nuevos enfoques de gestión a escala local (Femenia-Serra e Ivars-Baidal, 2018).

De esta manera se puede afirmar que el turismo responsable enlaza directamente con las consideraciones y criterios del turismo sostenible en materia de reducción de impactos ambientales, sociales y económicos en relación a los destinos de acogida e introduce el concepto de ética en el comportamiento tanto de los productores como de los consumidores (Rivera y Rodríguez, 2012). El mismo debe servir para mejorar la sostenibilidad del conjunto de la industria turística, pero establece una praxis específica que debe entenderse por la transformación de algunos elementos, tanto desde el punto de vista de la oferta como de la demanda (González Reverté, 2015).

Algunos de los cambios que está experimentando la demanda tienen estrecha relación con las buenas prácticas de turismo responsable, donde se destaca el perfil de un turista más experimentado y participativo, la realización de viajes más conscientes, que incorpora en su motivación el componente ético y formativo, desarrollo de vacaciones sostenibles y la búsqueda de una transformación interna durante su viaje. Las nuevas tendencias de los turistas, cuya satisfacción se centra en aspectos éticos y en la personalización del viaje, implican nuevos comportamientos y expectativas. Esta evolución de la demanda ha producido también una respuesta por parte de la oferta y han empezado a aparecer nuevos productos turísticos o productos mejorados donde la responsabilidad es un componente central de la visita.

En el ámbito nacional, Holguín es considerado uno de los destinos cubanos que ha iniciado el desarrollo de buenas prácticas del turismo responsable y ha logrado satisfacer, de esta manera, al consumidor. El mismo cuenta con más de 6000 habitaciones y 200 lugares de interés turístico, vinculados a la naturaleza y la cultura; así como un programa de desarrollo amplio y con más de 20 años en marcha, donde numerosas organizaciones públicas y privadas, turísticas y no turísticas participan en su ejecución. A partir de la 
importancia de la práctica del turismo responsable y las exigencias del consumidor turístico, la presente investigación se plantea como objetivo evaluar la influencia de las tendencias del comportamiento del consumidor turístico en el desarrollo responsable del destino Holguín, con el fin de conocer las limitaciones aún existentes para estar en capacidad de fortalecer las alianzas estratégicas en las comunidades, elevar la competitividad del destino y satisfacer a un mayor número de consumidores.

\section{Revisión de la literatura}

El turismo responsable es considerado un movimiento que busca establecer modelos de desarrollo turístico sostenibles y específicos para cada zona de destino, para lo que se deben tener en cuenta sus dimensiones. Diferentes autores han abordado estas dimensiones a través de variables e indicadores a considerar para evaluar estas prácticas. Entre ellos se encuentran: Rivera Mateus y Rodríguez García (2012), Guía Turismo Responsable Valencia (2018) y, Kastenholz y Galán-Ladero (2019). De la revisión de las propuestas anteriores fueron identificadas las variables comunes, las cuales se describen a continuación:

Variable socio-cultural: Considera el respeto a la autenticidad sociocultural de las comunidades anfitrionas, conservar sus activos culturales, arquitectónicos y monumentales, así como sus valores tradicionales, y contribuir al entendimiento y tolerancia interculturales.

\section{Indicadores}

- Oferta cultural

- Protección del patrimonio

- Bienestar de la comunidad

- Asociación local

- Desarrollo local

- Capacidad de carga

Variable medioambiental: Dar un uso óptimo a los recursos ambientales, que son un elemento fundamental del desarrollo turístico, manteniendo los procesos ecológicos esenciales y ayudando a conservar los recursos naturales y la diversidad biológica.

Indicadores

- Huella carbónica

- Gestión territorial

- Gestión del agua

- Gestión de la energía

- Gestión de los residuos

- Políticas de protección

Variable económica: Asegurar unas actividades económicas viables a largo plazo, que reporten a todos los agentes unos beneficios socioeconómicos bien distribuidos, entre los que se cuenten oportunidades de empleo estable y de obtención de ingresos y servicios sociales para las comunidades anfitrionas y contribuyendo a la reducción de la pobreza. 
Indicadores

- Prosperidad local

- Calidad de empleo

- $\quad$ Equidad social

- Satisfacción del visitante

- Impactos positivos

Variable tecnológica: La apropiación y uso de las soluciones tecnológicas contribuyen de manera efectiva a que las decisiones puedan tomarse en el mismo territorio turístico sobre una base empírica, toda vez que permite priorizar diversas acciones y medidas anticipando futuros escenarios, premisa fundamental para una gestión responsable del turismo y sus consecuentes impactos (Salessi, 2017). Asimismo, existe un número creciente de empresas en el sector turístico que adoptan una estrategia y una visión de responsabilidad social, abarcando los aspectos económicos, sociales, culturales y ambientales, fomentando un desarrollo sostenible (Alvarado, 2018) de su negocio y del destino del que forman parte.

Numerosos estudios han sido desarrollados en el entorno del turismo responsable (Rivera y Rodríguez, 2012; Rivera Mateos, 2015; González Reverté, 2015; Perogil Burgos, 2018 y (Femenia-Serra e Ivars-Baidal, 2018). Estas investigaciones relacionan buenas prácticas del desarrollo de turismo responsable en diferentes destinos. Estas prácticas denuncian los impactos negativos que el turismo convencional produce o puede producir en el futuro en las sociedades anfitrionas, así como la imagen distorsionada que las y los visitantes pueden hacerse de la realidad que han ido a conocer, además de que valora y reclama la responsabilidad de turistas, tour-operadores, anfitriones e instituciones públicas a la hora de favorecer modelos turísticos sostenibles.

Según plantea la Guía de Turismo Responsable de Valencia, los viajes de turismo responsable se caracterizan por:

- Difundir una forma de viajar que fomente el respeto a las culturas y la protección del medio ambiente, a la vez que pretende ser una herramienta de desarrollo para las comunidades de acogida.

- Aproximarse a otras culturas, de la mano de experiencias locales e indígenas. Los itinerarios combinan la visita a lugares interesantes, charlas sobre actualidad, historia y temas sociales con el conocimiento de experiencias locales ofertadas por organizaciones campesinas, civiles, ecologistas e indígenas.

- Viajar de manera responsable: una forma respetuosa de conocer la realidad de un país. La propuesta pretende fomentar un intercambio cultural entre viajeros y las comunidades de acogida en términos de igualdad y respeto.

- Ofrecer talleres de formación con anterioridad al viaje para que las y los viajeros adopten una actitud más activa, estando más y mejor informadas/os sobre la realidad del país que van a conocer.

- Fomentar los valores de viajar en grupo. El hecho de compartir experiencias tan intensas con los demás, favorece la sensibilización a través de las reflexiones 
colectivas. Además, incrementa la participación posterior en iniciativas relativas al voluntariado en el ámbito del turismo justo.

De esta manera, la expansión del turismo internacional podría generar modelos diferentes de desarrollo turístico, más equitativos, más equilibrados territorialmente, sostenibles, integrados, ecológicos, éticos, alternativos y solidarios que permitan, a través de diferentes modalidades o formas de hacer turismo, alcanzar metas de responsabilidad que prioricen el bienestar de las poblaciones anfitrionas sin perjuicio de la satisfacción de los turistas y consumidores. Los retos para mantener y ampliar las fronteras turísticas actuales pasan, en definitiva, por las 4Es: Ambiente (Environment), Energía, Ética y Educación (Rivera Mateus y Rodríguez García, 2012).

Por otra parte, el comportamiento del consumidor ha sido objeto de estudio de numerosas investigaciones (Serra Cantallops y Salvi, 2014; Tsai y Wang, 2017; Oter, 2018; Fetscherin, 2019; Pantano y Dennis, 2019 y Díaz-Meneses, 2019). El consenso general entre los autores que abordan el tema indica que el comportamiento del consumidor comprende los procesos implicados desde que los individuos o grupos de individuos buscan, seleccionan, adquieren, utilizan y evalúan productos, servicios, ideas o experiencias que ellos esperan que satisfagan sus necesidades o deseos. Es por ello que la mayoría de los modelos de comportamiento del consumidor consisten en tres etapas: pre-compra, consumo y post-consumo.

En la primera etapa los turistas potenciales reconocen la necesidad y sienten alguna motivación para viajar en sus vacaciones, buscan información sobre varios destinos y evalúan estas alternativas en orden seleccionar alguno. En esta elección influyen distintos factores, entre ellos, sicológicos, económicos, sociales, políticos, geográficos y demográficos, los cuales de manera global se pueden dividir en factores individuales y externos (Cohen, Higham, \& Cavaliere, 2011).

Durante la segunda etapa el turista experimenta el destino y sus productos y servicios. Esta etapa está compuesta por una serie de eventos y actividades que desarrolla el turista, así como el aprendizaje durante su estancia. En la última etapa, después de haber tenido sus vivencias de viaje, el consumidor evalúa sus experiencias, no solamente a partir de la información obtenida por las fuentes, sino también por sus propias expectativas. Esta evaluación se concreta en sentimientos de satisfacción o inconformidad con los productos o servicios consumidos, lo cual tiene consecuencias reales para la toma de decisiones futuras, en cuanto a retornar al destino o cambiar hacia otros destinos, sean domésticos o internacionales, además de trasmitir a otras personas los aspectos favorables o desfavorables de sus experiencias. De ahí la importancia de estudiar el comportamiento de los consumidores.

Se puede considerar que el comportamiento del consumidor turístico y sus tendencias actuales tienen una influencia significativa en el desarrollo del turismo responsable, al adoptar conductas responsables en el consumo de viajes que obligan a los destinos y organizaciones turísticas a adaptarse a los requerimientos de la demanda, las cuales deben ofrecer productos que preserven el medio ambiente, consideren el impacto social y el 
progreso económico de las partes involucradas. Entre las principales tendencias del comportamiento de los consumidores que influyen en el desarrollo del turismo responsable, identificadas a partir del análisis de diversas literaturas elaboradas por organizaciones turísticas internacionales y expertos del sector se pueden relacionar las siguientes:

- El desarrollo de viajes más conscientes

- El disfrute de vacaciones sostenibles

- El creciente interés por las culturas locales

- La búsqueda de transformación espiritual durante los viajes

- La influencia de las nuevas tecnologías para planificar los viajes

A partir de los estudios revisados se puede determinar existe un vínculo entre el comportamiento del consumidor y el desarrollo del turismo responsable, sin embargo, son insuficientes las investigaciones que abordan esta relación y sus impactos. Una evaluación realizada por Araña y León, 2018, indica los determinantes del comportamiento sostenible de los turistas en aspectos relacionados con decisiones que contribuyen a las emisiones de $\mathrm{CO} 2$ y al consumo de agua durante la experiencia turística. En el estudio se presentan resultados de dos experimentos relacionados con las decisiones de elección de emisiones de $\mathrm{CO} 2$ y de consumo de agua y energía, proporcionando evidencias acerca de la influencia que tienen los aspectos cognitivos y emocionales en las decisiones acerca de la elección de viajes turísticos con distintos niveles de emisión de $\mathrm{CO} 2$, y del ahorro de agua en los establecimientos de alojamiento. La evidencia aportada por ambos experimentos permite concluir que las políticas de precios a través de impuestos pueden ser tan relevantes como las políticas de sensibilización social y de provisión de información, así como la posible inducción de estados emocionales proclives a la adopción de decisiones responsables con el medio ambiente y la sostenibilidad (Araña y León, 2018).

\section{Metodología}

Diferentes autores han abordado las tendencias del comportamiento del consumidor relacionadas con el turismo responsable y la mayoría coincide en un conjunto de dimensiones relacionadas con los viajes conscientes, vacaciones sostenibles, interés por las culturas locales, el crecimiento espiritual y la influencia de las nuevas tecnologías. Para determinar las principales tendencias a partir de esas literaturas fue realizado un análisis de correlación, donde se empleó como escala de similitud binaria el método Jaccard. Este resultado evidenció un alto grado de centralidad entre los autores que abordan las principales tendencias del comportamiento del consumidor que influyen en el turismo responsable. Figura 1.

Para evaluar la relación que existe entre cada tendencia del comportamiento del consumidor y el turismo responsable se empleó el análisis del coeficiente Kendall a partir del criterio de expertos. Se solicitó a 10 expertos que valoraran la influencia de cada tendencia del comportamiento del consumidor en el turismo responsable, empleando una 
escala del 1 al 10 ( 1 totalmente en desacuerdo y 10 totalmente de acuerdo) teniendo en cuenta que es una de las más empleadas en las ciencias sociales.

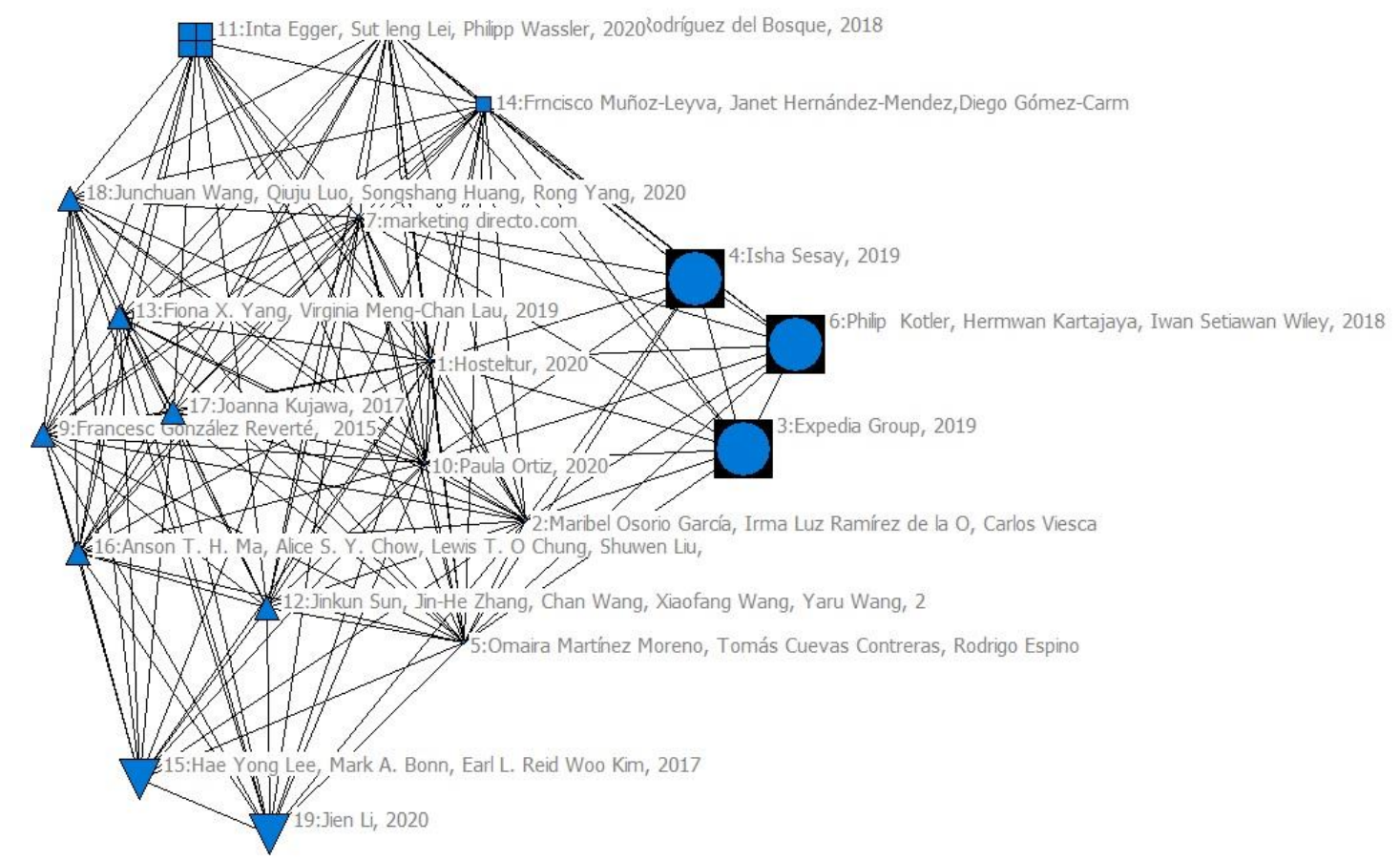

Figura 1. Autores que abordan las tendencias del comportamiento del consumidor que influyen en el turismo responsable.

El coeficiente de concordancia de Kendall (W) es igual a 0.816 indicando que los diez expertos, efectivamente concuerdan (coinciden en sus juicios en un 81,6\%). Habiendo existido concordancia entre los especialistas del tema, se verificó si dicha coincidencia era o no casual, con un nivel de significación del 5\%. Se desarrolló la prueba de hipótesis no paramétrica y como el valor de probabilidad de la prueba es igual a 0.000 entonces se cumple la región crítica y se rechaza la hipótesis nula. Esto indica que la concordancia entre los juicios emitidos por los expertos, es no casual. Además, el estudio permitió identificar las tendencias que más influyen en el desarrollo del turismo responsable: el desarrollo de viajes más conscientes, el disfrute de vacaciones sostenibles y el interés por las culturas locales, como se muestra en el gráfico 1.

Gráfico 1. Valoración promedio de los expertos. 


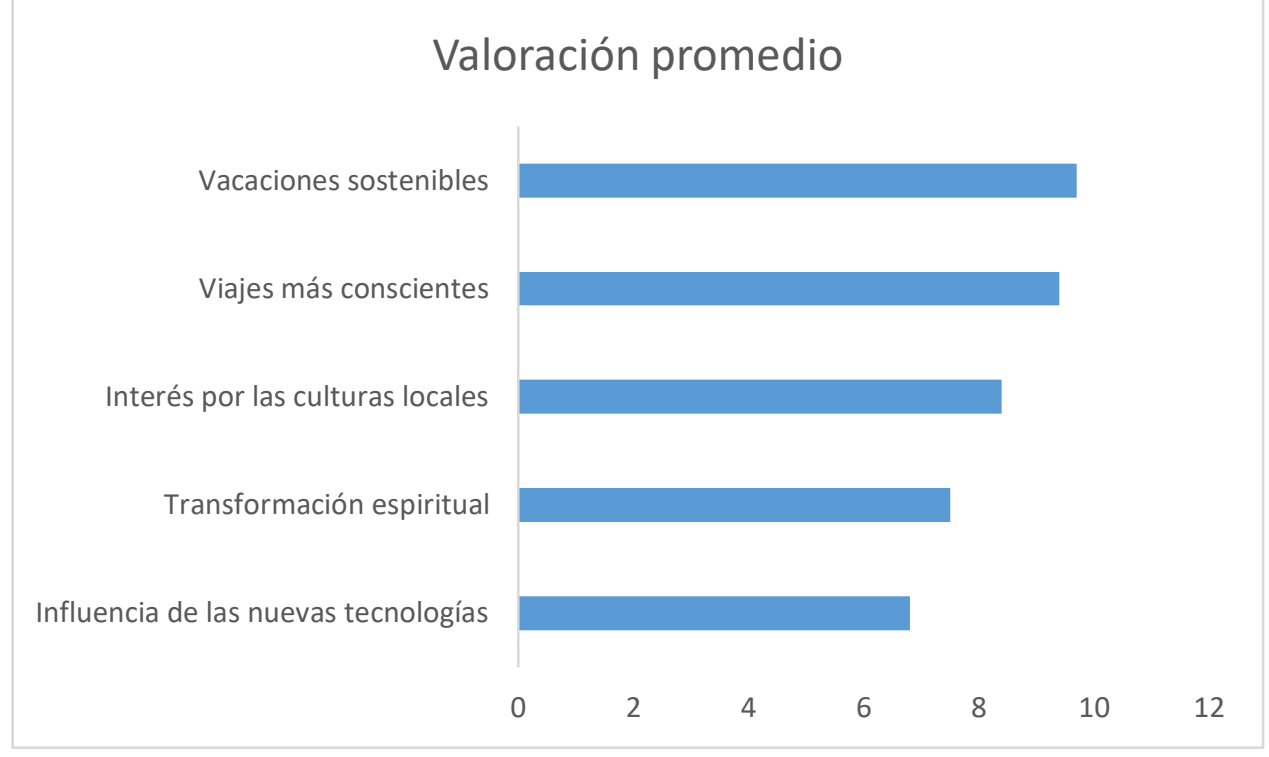

Fuente: Elaboración propia

En la investigación también fueron empleados otros métodos como la encuesta, para conocer los motivos de viaje y actividades que prefieren realizar los diferentes mercados que visitan el destino Holguín, la observación participante y las entrevistas a gestores turísticos del destino.

\section{Resultados y discusión}

Las relaciones entre las tendencias del comportamiento del consumidor y el desarrollo del turismo responsable en el destino Holguín se determinaron a partir de la aplicación del método de análisis de correlación. Entre los principales resultados se precisan las tendencias actuales del comportamiento del consumidor y cómo estas han impactado en el destino, favoreciendo las buenas prácticas de turismo responsable.

En primer lugar, el desarrollo de viajes más conscientes por parte de los consumidores ha generado un cambio en la forma de operar las organizaciones turísticas del destino. Esto se demuestra a partir de las nuevas políticas ambientales que han asumido las cadenas hoteleras, en función de conservar el medio natural. En entrevista realizada a directores de organizaciones turísticas del polo Guardalavaca se identificó que algunas de las estrategias tomadas en Holguín se corresponden con solicitudes que han realizado a las instalaciones clientes fieles y líderes de opinión de algunos segmentos de mercado. Una evidencia de ello es la iniciativa tomada en el Hotel Brisas Guardalavaca, denominada la siembra del árbol, actividad propuesta por un grupo de clientes repitentes del hotel en aras de reforestar algunas áreas que fueron afectadas por un fenómeno climatológico. La iniciativa se ha mantenido con el transcurso de los años.

Por otra parte, las cadenas hoteleras Sol Meliá e Iberostar han adoptado políticas de cambio en la cultura corporativa y está arrojando resultados reales para la salud del planeta. La idea de Iberostar de sustituir vasos desechables por reutilizables ha conseguido eliminar miles de kilogramos de emisiones de $\mathrm{CO} 2$ cada año, así como los amenties que se envuelven en materiales biodegradables. Con estos pasos proactivos en 
la reforma de la política de la empresa no solo los empleados adoptan enfoques ambientales más sensatos, sino que se transmite a sus consumidores.

El disfrute de vacaciones sostenibles es otra de las tendencias de los consumidores turísticos que ha influido en la cartera de productos del destino Holguín. A partir del creciente interés de los clientes por consumir productos y servicios ecológicos las agencias de viajes del destino han rediseñado sus ofertas, prestando especial atención a las experiencias rurales. Un análisis realizado sobre los catálogos de productos de las agencias de viajes durante el período 2015-2020 demostró el incremento en el número de excursiones vinculadas a la naturaleza y la cultura autóctona de los destinos, así como el diseño de viajes a la medida. Actualmente, dos de las agencias de viajes más importantes del destino Holguín, Cubanacán y Cubatur, quienes representan alrededor del $65 \%$ de las ventas de excursiones, poseen en su cartera de productos para 2020 más del 70\% de las ofertas relacionadas con la naturaleza y la identidad cultural de las comunidades, como se muestra en el gráfico 2.

\section{Gráfico 2.}

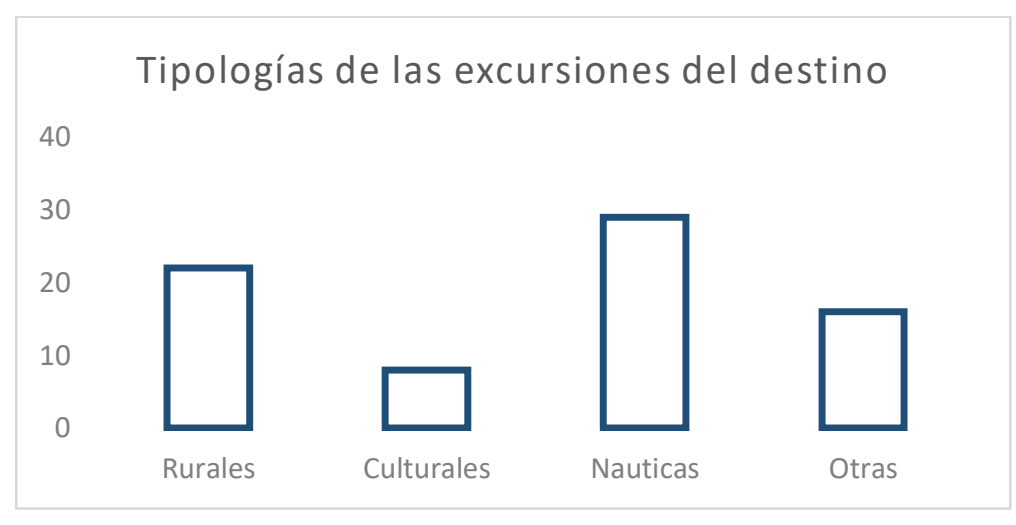

Fuente: Elaboración propia

Las 64 excursiones presentes en el catálogo de estas agencias reportaron un total de 47366 clientes que disfrutan de esas ofertas durante su estancia en el año 2018 en el destino. Además, se identifica como tendencia el aumento de la compra de excursiones relacionadas con la naturaleza y la cultura autóctona, alcanzando la cifra de 37728 pax, lo cual representa un $79 \%$ del total de clientes que compraron excursiones en dicho año, como se detalla en el gráfico 3.

\section{Gráfico 3.}


Ventas de excursiones en el destino

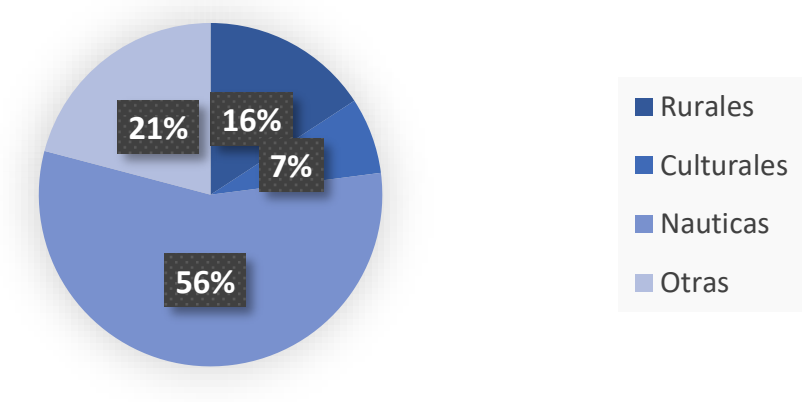

Fuente: Elaboración propia

Por otro lado, la importancia que han tomado las culturas locales para los consumidores se puede evidenciar desde el interés que manifiestan los clientes por conocer destinos donde puedan aprender de las comunidades en las que se enclavan los productos turísticos y a su vez relacionarse con las mismas. En una encuesta aplicada a canadienses y alemanes que visitan el destino Holguín, se puede determinar que el principal motivo de viaje de los clientes canadienses es el descanso y relax (65.5\%), sin embargo, también refirieron como motivos de viajes: conocer nuevas culturas $(27.6 \%)$, conquistar la felicidad $(3.5 \%)$ y encontrarse con la naturaleza $3.4 \%$. A su vez, su actividad favorita es el disfrute del sol y la playa con un $50.26 \%$, además de ello los clientes encuestados refirieron preferencias adicionales como: pasear en catamarán 14.63\%, asistir a espectáculos culturales diversos $11.29 \%$, caminar por la cuidad $10.02 \%$, disfrutar de comida típica campesina $8.79 \%$ y visitar lugares históricos $5.01 \%$.

Por su parte, de los clientes alemanes que visitan el destino, el 58,3\% de los encuestados refiere que busca encontrarse con la naturaleza, seguidos por el $25 \%$ que busca relajarse, el $12,5 \%$ conocer nuevas culturas y el $4.2 \%$ conquistar la felicidad. En cuanto a las actividades que realizan en el destino se identifican, en orden de preferencia, intercambiar con pobladores locales, recorrer senderos naturales, visitar lugares históricos, conocer sitios arqueológicos pescar y caminar por la ciudad. En resumen, se puede afirmar que entre los motivos de viaje de estos segmentos de mercado resalta el interés por conocer la cultura local y el intercambio con la naturaleza y entre las actividades que prefieren realizar predomina la presencia de atractivos naturales y culturales auténticos del destino.

La búsqueda de la transformación espiritual durante los viajes es considerada otra de las motivaciones que tienen en cuenta los consumidores, quienes buscan vincularse a las actividades que se desarrollan en las comunidades locales y en las propias instalaciones para preservar el medio ambiente. Un ejemplo de ello es en el polo turístico holguinero el hotel Brisas Guardalavaca, quien implementa desde el año 2003 el Proyecto "Mi aporte al Medio Ambiente", que surge con el objetivo de dar continuidad a la actividad de jardinería y contribuir con la siembra de árboles en áreas que lo necesiten. Estas actividades se realizan con la participación de clientes y miembros de la comunidad. La actividad posibilita disminuir la falta de sombra en la playa y solucionar afectaciones ocasionadas por eventos meteorológicos extremos. 
La instalación desarrolla otras actividades comunitarias, fundamentalmente con niños y jóvenes, trabajadores y clientes, los cuales que inciden en el cuidado y conservación del entorno. Entre las actividades preferidas por los clientes que visitan dicho hotel se encuentra la siembre del árbol, la cual se realiza semanalmente en la instalación y donde participan los clientes repitentes. Cada semana se siembra un árbol en las áreas verdes del hotel, ratificando de esta manera el compromiso de los clientes con el medio ambiente. Alrededor del 22\% del total de clientes al cierre del año 2019 participó en dicha actividad. Estas actividades voluntarias reflejan la conciencia ambiental que han adquirido tanto los consumidores como los prestatarios de servicio.

La influencia de las nuevas tecnologías ha generado cambios en el comportamiento de los consumidores y a su vez, en las estrategias que han trazado las entidades turísticas para satisfacer las demandas. En el destino Holguín se han realizado diversas mejoras tecnológicas durante los últimos años, entre ellas la instalación de sistemas de clima centralizados, que garantizan una mejor eficiencia energética y también poseen un sistema de recuperación de calor, el cual apoya el calentamiento del agua de las habitaciones y permite de esta forma disminuir el consumo de gas que se emplea en esta actividad. Se ha logrado en un elevado porciento sustituir las luminarias fluorescentes por Led, que consumen un 50\% menos de energía. Además, para mejorar la accesibilidad a internet, se ha instalado la red WIFI en la mayoría de las instalaciones turísticas del destino, que facilita a los clientes una mayor conectividad y está prevista la automatización progresiva de sistemas hoteleros para un mayor control y uso sostenible de los recursos energéticos.

Por otra parte, fue realizada una evaluación global de los indicadores del turismo responsable en el destino turístico Holguín, la cual arrojó los resultados siguientes:

1. Variable sociocultural

La oferta del destino turístico Holguín incluye un numeroso grupo de atractivos culturales, como eventos, sitios emblemáticos relacionados con costumbre y tradiciones, las propias tradiciones, grupos artísticos, etc.

Un ejemplo de ello son los eventos culturales propios y de gran convocatoria nacional e internacional como Las Romerías de Mayo, que, a través del arte joven, recrea las mejores tradiciones y que acoge al Festival Mundial de Juventudes cada año; así como la Fiesta de la Cultura Iberoamericana. También están presentes otras manifestaciones y agrupaciones artísticas como: Teatro Lírico y CODANZA, importantes exponentes de la pintura y la literatura, entre otras.

Otro atractivo significativo es el reconocimiento de la ciudad de Holguín como la Ciudad de los Parques, por la presencia de varios de estos espacios dedicados al esparcimiento de su población y visitantes. Destacan entre ellos el parque Calixto García, centro cultural y comercial de la ciudad; el parque Carlos Manuel de Céspedes (San José) y el parque de Las Flores. Los mismos están rodeados de numerosos lugares que atraen a los turistas como: Museo La Periquera, de singular valor arquitectónico e histórico, declarado monumento nacional; Museo de Historia Natural; Catedral San Isidoro, de estilo 
neoclásico, donde se ofició la misa fundacional de la ciudad en 1720; mural Orígenes, obra escultórica que marca el lugar fundacional de la ciudad; Casa Editorial Cuadernos Papiro, donde se editan libros con papel manufacturado de gran calidad y autenticidad elaborado de forma artesanal, entre otros muchos.

La protección al patrimonio natural y cultural es un principio en Cuba y en Holguín y se refleja en la conservación de monumentos arquitectónicos, de sitios naturales de valor, del respeto a costumbre y tradiciones.

También se busca el bienestar de las comunidades vinculadas con el desarrollo del turismo, a partir de considerar el beneficio a las mismas con infraestructuras desarrolladas en polos turísticos cercanos, preferencia para el empleo en el sector, todo lo cual favorece el desarrollo local y que los residentes se involucren y perciban el turismo de manera favorable.

\section{Variable medioambiental}

En el mundo el turismo de masas se ha mostrado especialmente agresivo con el medio ambiente, sobre todo por la urbanización de zonas naturales sin tener en cuenta su integración en el paisaje, la sobreexplotación del recurso agua, los problemas relacionados con el tratamiento de residuales y la contaminación del aire por el uso de vehículos. En Holguín varios de estos temas tienen un tratamiento diferente, resultado de las políticas que sigue Cuba en esta materia.

Cuba firmó el Convenio para la Declaración de la Zona de Turismo Sustentable del Caribe, considerando en el mismo la calidad de los cuerpos de aguas marinas, índice de consumo energético, conservación del ecosistema, financiamiento en la gestión y protección ambiental. El MINTUR de conjunto con el CITMA desarrolló una propuesta de indicadores para el país para cumplir los compromisos asumidos, cuestión que se viene cumpliendo en el destino Holguín y que se expresa en:

El programa de desarrollo materializado y proyectado prevé la conservación de los ecosistemas. Un ejemplo de ello es la construcción de hoteles cercanos a la playa que se insertan en la vegetación de forma armónica, preservando la misma y realzando sus valores.

En los polos turísticos se realiza una gestión del agua que considera el reciclaje de la misma para usos como la jardinería, cultivos y otros, con el fin de optimizar su uso; así mismo los residuos sólidos reciben tratamiento y nuevos usos. Aunque las redes de acueducto y alcantarillado no alcanzan a beneficiar el total de la población de la provincia, si están completos en los desarrollos turísticos y las comunidades aledañas, además los gobiernos locales despliegan el servicio en ciudades y el resto de las localidades.

La gestión para disminuir el índice de consumo energético aún es insuficiente, aun cuando se impulsan un conjunto de medidas. Aún no se desarrollan fuentes de energía renovable vinculadas a los desarrollos turísticos del destino. Tampoco se avanza lo necesario en la reducción de la huella de carbono.

3. Variable tecnológica 
Entre las tecnologías de mayor impacto en la actualidad están las de la información y las comunicaciones. En el destino Holguín el sistema de información y promoción turística, liderada por INFOTUR, tiene como cualidades la presencia en Facebook, Twitter y YouTube; así como en el portal holguintravel. Se interactúa con los clientes únicamente en Facebook y solo existe accesibilidad a información pública a través de dispositivos inteligentes dentro de Cuba en las zonas con conexión a Internet. No se gestiona adecuadamente la reputación on line en los sitios de opinión y no existe una plataforma tecnológica integrada de datos para la gestión del destino.

Por otra parte, se avanza en la implementación del Gobierno electrónico y aunque existe una limitada disponibilidad financiera para el desarrollo de una gobernanza electrónica plena, el Gobierno Central ha dado una alta prioridad para esta actividad en el corto y mediano plazo, por lo cual este indicador se debe transformar favorablemente.

\section{Variable económica}

La provincia de Holguín posee superhábit (más ingresos que gastos) hace varios años en su desempeño económico, a lo cual ha contribuido decisivamente el desarrollo del turismo en el destino, lo que ha influido favorablemente en la prosperidad de la población, especialmente en los asentamientos vinculados a zonas turísticas. Las personas empleadas en el sector del turismo en el territorio poseen numerosos beneficios, siendo este uno de los principales impactos positivos que genera.

Los resultados de satisfacción del cliente en el destino son los mejores del país, de acuerdo a la encuesta que aplica INFOTUR regularmente en los diferentes destinos, siendo este un elemento importante para el desarrollo de un turismo responsable y una prueba que Holguín trabaja en esta dirección ya que la demanda actual apuesta por un consumo responsable.

Como resultado del análisis de las variables se puede apreciar que existen algunas insuficiencias en el destino para el desarrollo de buenas prácticas del turismo responsable, sin embargo, predominan las iniciativas encaminadas al desarrollo de un turismo responsable, que ya poseen resultados concretos, algunos que responden a políticas nacionales y otros aportados por el territorio.

Entre estas iniciativas, aplicadas sobre todo al ámbito de la gestión comercial, se pueden mencionar las variantes de concepción de productos que responden al agroturismo, propuesta que considera el turismo como factor de desarrollo económico y social y promueve la interrelación entre una agricultura sostenible y un turismo solidario, convirtiendo a los campesinos en actores de su propio desarrollo y fomentando proyectos e iniciativas para luchar contra la desertización de las zonas rurales.

El ecoturismo también se desarrolla en el destino. El mismo tiene una relación directa con la salud humana y la salud de los ecosistemas y se asocia habitualmente a ofertas en países lejanos por sus características de territorios no desarrollados o espacios vírgenes, pero también tiene muchas posibilidades en el destino Holguín como oferta turística que considera la conservación del patrimonio cultural, la biodiversidad rural y natural, la 
producción agraria ecológica, entre otros. Aunque existen diferentes interpretaciones, en general el turismo ecológico se promueve como un turismo "ético", en el cual también se presume como primordial el bienestar de las poblaciones locales y tal presunción en general se refleja en la estructura y funcionamiento de las empresas que ofrecen el servicio.

Existen alojamientos responsables en Pinares de Mayarí y Cayo Saetía, situados en entornos naturales en los que se desarrollan actividades enmarcadas en el senderismo, ciclo turismo y otros deportes relacionados con el respeto al medio ambiente.

\section{Conclusiones}

Como resultado del trabajo se arribó a las conclusiones siguientes:

- Se determinaron las principales tendencias del comportamiento del consumidor turístico que influyen en el desarrollo del turismo responsable, donde se destaca el desarrollo de viajes más conscientes, el disfrute de vacaciones sostenibles, el interés de los consumidores por las culturas locales, la búsqueda de la transformación espiritual durante los viajes y la influencia de las nuevas tecnologías.

- Se realizó un análisis de la influencia de estas tendencias en el destino turístico Holguín, donde se demostró las buenas prácticas de turismo responsable asumida por las organizaciones del destino para satisfacer la demanda y contribuir de esta manera a la preservación del planeta.

- Fueron identificados los principales indicadores de turismo responsable donde el destino Holguín posee buenas prácticas: la oferta cultural; la protección del patrimonio; el desarrollo local; la gestión territorial; la gestión del agua y los residuos; las políticas de protección; la prosperidad local; calidad de empleo; satisfacción del visitante y los impactos positivos y por otra parte los indicadores cuya gestión debe mejorar el destino Holguín se concentran en: gestión de la energía; huella carbónica y gestión de las tecnologías de la información y las comunicaciones.

\section{Referencias bibliográficas}

Alvarado, R. (2018). Ciudad inteligente y sostenible: hacia un modelo de innovación inclusiva. Revista de Tecnología y Sociedad, vol 13. Recuperado de: http://www.scielo.org.mx/pdf/prts/v7n13/2007-3607-prts-7-13-00002.

Araña, J. y León. Carmelo. (2018). Comportamiento del consumidor y turismo sostenible. Cuadernos Económicos de ICE. Nº3, pp. 46-68.

Arrans, A. (2019). ¿Cuáles son las tendencias turísticas para 2020? Centro Europeo de Postgrado. 
Cohen, S. A., Higham, J. E. S., \& Cavaliere, C. T. (2011). Binge flying: Behavioural addiction and climate change. Annals of Tourism Research, 38(3), 1070-1089. doi:https://doi.org/10.1016/j.annals.2011.01.013

Díaz-Meneses, G. (2019). A multiphase trip, diversified digital and varied background approach to analysing and segmenting holidaymakers and their use of social media. Journal of Destination Marketing \& Management, 11, 166-182. doi:https://doi.org/10.1016/j.jdmm.2017.07.005

Femenia-Serra, F. \& Ivars-Baidal, J. A. (2018). Smart tourism: Implicaciones para la gestión de ciudades y destinos turísticos. En M. T. Cantó López, J. A. Ivars Baidal, \& R. Martínez Gutiérrez (Dir.), Gestión inteligente y sostenible de las ciudades: Gobernanza, smart cities y turismo (pp. 129-151). Valencia: Tirant Lo Blanch.

Fetscherin, M. (2019). The five types of brand hate: How they affect consumer behavior. Journal of Business Research, 101, 116-127. doi:10.1016/j.jbusres.2019.04.017

Galán-Ladero, M. \& Kastenholz, E. (2009). Turismo accesible como ejemplo de responsabilidad social en las empresas y destinos turísticos. El caso de Lousã (Portugal).

González Reverté, F. (2015). Caracterización de la oferta comercializada bajo la etiqueta de turismo responsable en España. Boletín de la Asociación de Geógrafos Españoles No 67, pp. 189-212.

Guía de Turismo Responsable de Valencia. (2018).

López, A., Lancis, E., García, S., Alcantud, A., García, B. \& Muñoz, N. (2015). Informe destinos turísticos inteligentes: construyendo el futuro.

Organización Mundial del Turismo (2009). Conceptos y Definiciones. Desarrollo Sostenible del Turismo.

Organización Mundial del Turismo (2015). Manual sobre turismo accesible para todos: principios, herramientas y buenas prácticas.

Organización Mundial del Turismo (2020). Barómetro OMT.

Oter, Z. (2018). Hospitality marketing and consumer behavior: Creating memorable experiences. Journal of Hospitality and Tourism Management, 36, 111-112. doi:10.1016/j.jhtm.2017.10.002

Pantano, E., \& Dennis, C. (2019). Store buildings as tourist attractions: Mining retail meaning of store building pictures through a machine learning approach. Journal of Retailing and Consumer Services, 51, 304-310. doi:https://doi.org/10.1016/j.jretconser.2019.06.018 
Perogil Burgos, J. (2018). Turismo solidario y turismo responsable, aproximación a su marco teórico y conexiones con la inteligencia turística. Revista Iberoamericana de Economía Solidaria e Innovación Socioecológica. Vol. 1, pp. 23-48.

Rivera Mateus, M. \& Rodríguez García, L. (2012). Turismo responsable, sostenibilidad y desarrollo local comunitario.

Ruano, D. \& Rojas, P. (2006). Marcas responsables: ¿oportunidad o necesidad? Harvard Deusto Marketing y Ventas, $N^{\circ} 77$, pp. 40-43.

Rivera Mateos, M. (2015). Hacia nuevas formas de turismo, responsable, intercultural y experiencial. Revista Turismo y Desarrollo, No 15, pp. 4-9.

Salessi, M. L. (2017). Destinos Turísticos Inteligentes: Una perspectiva desde la inclusión social y la participación comunitaria. Actas del Seminario Internacional Destinos Turísticos Inteligentes: nuevos horizontes en la investigación y gestión del turismo. Universidad de Alicante, pp 215 - 227.

Serra Cantallops, A., \& Salvi, F. (2014). New consumer behavior: A review of research on eWOM and hotels. International Journal of Hospitality Management, 36, 41 51. doi:10.1016/j.ijhm.2013.08.007

Tsai, C.-T., \& Wang, Y.-C. (2017). Experiential value in branding food tourism. Journal of Destination Marketing \& Management, 6(1), 56-65. doi:https://doi.org/10.1016/j.jdmm.2016.02.003 


\section{PARA CITAR EL ARTÍCULO INDEXADO.}

Pérez Ricardo, E. del C., Medina Labrada, J. R., \& Fernández Cueria, N. (2021). Influencia de las tendencias del comportamiento del consumidor turístico en el desarrollo responsable del destino Holguín. Explorador Digital, 5(1), 133-151. https://doi.org/10.33262/exploradordigital.v5i1.1494

\section{Ciencia \\ LDigital}

El artículo que se publica es de exclusiva responsabilidad de los autores y no necesariamente reflejan el pensamiento de la Revista Explorador Digital.

El artículo queda en propiedad de la revista y, por tanto, su publicación parcial y/o total en otro medio tiene que ser autorizado por el director de la Revista Explorador Digital.
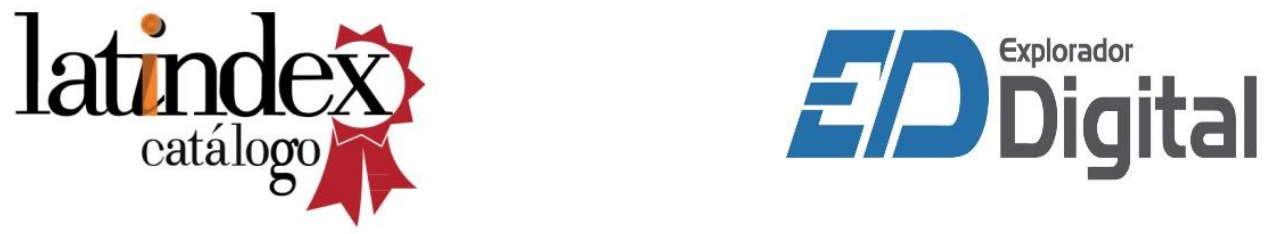